\title{
Towards Non-invasive Adaptive Meta-Cognitive Support for Online Training
}

\author{
Adam Moore $^{1}$, Owen Conlan ${ }^{1}$, Declan Dagger ${ }^{2}$, Vincent Wade ${ }^{1}$ \\ ${ }^{1}$ KDEG, School of Computer Science and Statistics \\ Trinity College, Dublin \\ Dublin 2, Republic of Ireland \\ \{mooread,oconlan,vwade\}@scss.tcd.ie \\ ${ }^{2}$ EmpowerTheUser (ETU) Ltd. \\ Trinity Technology \& Enterprise Campus, The Tower, \\ Pearse Street, Dublin 2, Republic of Ireland \\ declan.dagger@empowertheuser.com
}

\begin{abstract}
- online training is an increasingly important facet of the skills enhancing portfolio of any organization and has an especial place within life-long learning, career development and other self-regulated adult learning. We here present an overview of work towards developing online training, which is augmented by contextual cues; training which is personalized and adapted, in real-time, towards the learning objectives of the user, whilst supporting a wide range of meta-cognitive activities. This work brings technologies developed for the fantastical scenarios of serious gaming into the 'real world' we also begin to address some of the underlying challenges in this transition.
\end{abstract}

Keywords - real-time, adaptation, contextually aware, metacognition, non-invasive, personalisation, Digital Electronic Games, Training, multi-adaptive

\section{INTRODUCTION}

Increasingly, organizations are deploying technologymediated, (especially web-based) training materials. Currently, these materials do a very good job of teaching basic skills, however, the equally important part of preparation for the deployment of these skills in an operationalised environment can be considered to be lacking. Good training has value in providing opportunities for reflection, and other types of metacognition, allowing self-evaluation, self-awareness and self-pacing and other socalled soft skills. It is also important to have training that can give hints as to reality of the situation skills might be employed within - emotions that might be felt, off-task issues, etc. These should help construct the fidelity of the training experience, without detracting from the cohesion and narrative flow [1] of the training material. Current technologies which have some kind of support for reflection or other metacognitive activities [2] often do this in a manner discontinuous with the training material - in a similar way that early education games often contained crude switches, Shavian reversals [3], between gaming and learning content.

There exists a real challenge with the transition of application from games to training where the fidelity to the real-world experience is critical - it being necessary to maintain narrative flow whilst still finding areas to 'inject' support, in a real-time, non-invasive manner that builds on an awareness of both the needs of the learner's interaction with the learning material and the creation of an experience aligned to the deployment of those skills.

In this paper we will propose an approach, to be realized as part of the FP7 ImREAL project [http://www.imrealproject.eu/], to address these challenges by applying the techniques developed in two separate fields: real-time noninvasive adaptation and affective metacognitive modeling.

\section{NON-INVASIVE ADAPTATION IN DEGS}

Digital Electronic Games (DEGs) provide opportunities to shape experiential encounters with learning materials in an immersive, highly motivating way. Current DEGs are providing real-time, non-invasive adaptation to the learning requirements of those playing the games.

This adaptation can occur at two levels - the macro and the micro - and may be delivered as a service that sits alongside the Game Engine, allowing interventions to be delivered that are both apart from the development cycle of the main game, whilst at the same time being contextually sensitive to the ongoing in-game activity. For example, the Adaptive Learning In Games through Non-invasion (ALIGN) [4] system provides adaptive axes such as Cognitive feedback, Meta-cognitive feedback, Affective/motivational feedback, Knowledge-based hinting and Progression hinting. ALIGN was developed out of work developing adaptive engines for DEGs (such as ELEKTRA and 80Days) that allowed real-time, non-invasive support for the learner through consuming semantically rich information provided by the game engine.

\section{METACOGNITIVE SCAFFOLDING SERVICES}

Both ELEKTRA and 80Days model the progression of the learner's competency with regard to the learning objectives. However, an important part of any learning process is the skills around learning itself. This process of thinking about thinking, metacognition, along with an awareness of the affective state, or sentiments of a learner, provide a useful feedback loop in judging the motivation and progress of the student. At the same time, the model of these characteristics allows feedback to be appropriately contextualized to the learner.

In order to support metacognitive processes, the Goby service has been developed. This takes a symbiotic-dialog approach positioning itself as a third party service working 
with training software by engaging in a dialog with the learner in order to promote a particular skill through selfreflection. Goby implements ETTHOS (Emulating Traits and Tasks in Higher-Order Schemata) [6], which is focused on the representation of useful cognitive thought. Prompts implicitly update the model, while answers to questions explicitly update it.

\section{INTEGRATING ADAPTATION \& SCAFFOLDING FOR TRAINING}

It's important that when enabling these adaptive services to augment learning through training simulations we leverage the connection with the real world, providing affective support, both by inferring the state of the learner and providing cues as to what the learner may expect as they encounter the situations they are being trained for.

The challenge, then, is to maintain the immersive experience of the training simulation, whilst at the same time providing timely and contextually sensitive interventions allowing metacognition. In DEGs this is somewhat easier as the scenario (world, if you will) can be constructed and shaped in order to serve this goal. An effective training simulation is entirely antithetical to this approach - it is essential to the success of the training that it has the utmost fidelity to the situation it is providing training for.

We will do this by delivering a new open framework of intelligent services that can be plugged in to simulated environments to enhance self-regulated learning, that is, we will not create a tailored component as part of one particular system, but rather deliver a distributed architecture, able to be contextually sensitive by an informed awareness of the semantic content and a competence model of the learner's metacognitive progression and affective state.

\section{USE CASE}

We propose implementing these services to provide affective and reflective support to trainees using video simulations of interviews to learn how to diagnose mental illnesses. Currently, the trainees are presented with a virtual, adaptive interview in which the questions they pose can lead to a variety of outcomes. The simulator plays a short clip and then provides a set of responses for each stage of this process. The videos branch according to the responses chosen by the trainee.

At discrete stages the trainee is asked to assess and reflect on their performance, specifically in the areas of Orientation to consult; Rapport building; Symptom elicitation; Communicating treatment plan; and Follow up procedures. The assessment is in the form of their projection of how successful they will be. For example, a trainee may, based on their current knowledge and confidence, project an $85 \%$ success in rapport building. As they go through the simulation the system reports their actual success. Based on the correspondence or difference between the reported and projected success the trainee is asked to reflect on their performance.

The metacognitive service will generate a personalized reflection prompt that utilizes information about the questions the trainee posed and information from a Real World Model [7]. At each stage of reflection these prompts will be tailored to the trainee's performance, allowing them to more accurately assess their capabilities and potential shortcomings. These prompts will be presented in a consistent, contextually sensitive and non-invasive manner and will utilize appropriate affective and meta-cognitive models. It is envisaged that this will lead to more effective planning and self-assessment of skills.

\section{CONCLUSIONS}

In conclusion, there is real potential for a step-change in the efficacy and fidelity of electronically mediated training by deploying solidly tested technologies for real-time adaptation and personalization at the micro and macro level, leading to metacognitive skill development (e.g. so-called soft skills, learning to learn) and built-in affordances for reflection. Moving out of the fantastical world of serious gaming brings challenges in shaping the user experience, changing in focus from the ability to create a world based on the learning scenario and objectives, to creating an experience with direct integrity to the 'real world' built from training material, with additional scaffolding seamlessly integrated as a part of that experiential flow.

We will take the real-time non-invasive methodologies of ALIGN, as explored in ELEKTRA and 80Days and use them to deliver the currently non-contextualized metacognitive scaffolding provided in Goby, examining the usefulness of the ETTHOS model as a first step in modeling the metacognitive processes of the learner accessing training material.

\section{ACKNOWLEDGMENT}

The research leading to these results has received funding from the European Union Seventh Framework Programme (FP7/2007-2013) under grant agreement number ICT 257831 (ImREAL project).

\section{REFERENCES}

[1] M. Csikszentmihalyi, "Flow: The psychology of optimal experience." NY: New York: Harper and Row., 1990.

[2] C. Gama, "Metacognition in Interactive Learning Environments: The Reflection Assistant Model. Intelligent Tutoring Systems" 3220, pp. 668-677, 2004.

[3] S. Papert, "Does Easy Do It? Children, Games, and Learning," Game Developer, vol. 5, p. 88, 1998.

[4] N. Peirce, O. Conlan, and V. Wade, "Adaptive Educational Games: Providing Non-invasive Personalised Learning Experiences,", Proceedings of the Second IEEE International Conference on Digital Games and Intelligent Toys Based Education, 2008, pp. 28-35.

[5] O. Conlan, C. Hampson, N. Peirce, and M. Kickmeier-Rust, "Realtime Knowledge Space Skill Assessment for Personalized Digital Educational Games," Ninth IEEE International Conference on Advanced Learning Tech, Riga, Latvia: IEEE, 2009, pp. 538-542.

[6] V. Macarthur and O. Conlan, "Using Psychometric Approaches in the Modelling of Abstract Cognitive Skills for Personalization," Proc. Lifelong User Modelling Workshop (LLUM 09), 2009, pp. 22-26.

[7] Wichai Eamsinvattana, Vania Dimitrova, David Allen. 2009. Personalised Support for Reflective Learning in Fire Risk Assessment. In Proc. Conf. on Artificial Intelligence in Education, IOS Press, Amsterdam, The Netherlands, pp. 765-766 